T1 MATRIX METALLOPROTEINASE-DRIVEN TISSUE DESTRUCTION IN HUMAN TUBERCULOSIS (TB) IS MEDIATED BY TH-17 CYTOKINES AND THE PI3K/P110A/ P70S6K CASCADE

\author{
doi:10.1136/thoraxjnl-2011-201054a.1
}

${ }^{1} \mathrm{~S}$ Singh, ${ }^{2} \mathrm{U}$ K Singh, ${ }^{1} \mathrm{P}$ T Elkington, ${ }^{1} \mathrm{~J} S \mathrm{~S}$ Friedland. ${ }^{1}$ Imperial College London, London, UK; ${ }^{2}$ Nalanda Medical College and Hospitals, Patna, India

Mycobacterium tuberculosis (Mtb) kills 1.7 million people annually. The Th1 paradigm does not explain TB-driven cavitation. Current treatment is lengthy with many adverse effects. The Interleukin-23/ Th17 axis plays a critical role in early Mtb containment. Respiratory stromal cells are important first-line defence and secrete Matrix metalloproteinases (MMPs). Role of MMPs, which are substratespecific proteases causing extracellular matrix degradation/remodelling, was investigated in TB. Human bronchoalveolar lavage (BAL) samples from 35 well-characterised $\mathrm{TB}$ and control patients were analysed for MMPs and Th17 cytokines. TB/Control lung biopsies were stained for MMPs/IL-17. Primary normal human bronchial epithelial cells (NHBEs) and MRC-5 fibroblasts were stimulated with IL-17/IL-22/IL-23, alone and in combination with conditioned medium from Mtb-infected monocytes (CoMTb). Secretion, gene expression, gene silencing, intracellular signalling were investigated by luminex, ELISA, zymography, dual-luciferase promoter-reporter, realtime RT-PCR, siRNA transfection. MMPs were up-regulated in Human TB BALs $(\mathrm{p}<0.0001)$. This positively correlated with cavitation score on CXRs. TIMPs (tissue inhibitor of metalloproteinase) and IL-17/IL-23 were unaltered but IL-22 was increased in TB BAL. IL-17 and MMP-3 were co-expressed in pneumocytes around granulomas in TB lung biopsies. CoMTb (but not direct infection) upregulated secretion and gene expression of MMP-1 (collagenase, $\mathrm{p}<0.0001$ ), MMP-3 (stromelysin, $\mathrm{p}<0.001$ ) and MMP-9 (gelatinase, $\mathrm{p}<0.0001$ ) from NHBEs. MMP-3 protein and promoter activity in MRC-5s was also increased $(p<0.001)$. AKT inhibition suppressed all MMPs $(p<0.01)$ whereas siRNA and chemical inhibition of the proximal PI3Kp110a subunit abrogated MMP-3 only $(p<0.001)$. Distally, p70S6K (mTOR) blockade with rapamycin abrogated TBdriven MMP-1 and MMP-3 $(\mathrm{p}<0.001)$. MMP-9 production was unaffected by proximal/distal inhibition of PI3K.IL-17 independently and also synergistically with CoMTb augmented MMP-3 secretion/gene expression from NHBEs and MRC-5s in a dosedependent manner (peak $8 \mathrm{ng} / \mathrm{ml}, \mathrm{p}<0.0001$ ). This was p38dependent, confirmed by p38-specific siRNA. In contrast, IL-17 down-regulated CoMTb-driven MMP-9 to baseline $(p<0.01)$. IL-22 augmented MMP-3 from fibroblasts but not from NHBEs. IL-23 did not drive MMPs.

Summary MMPs are key mediators of tissue damage in human pulmonary $\mathrm{TB}$ and are regulated in a cell- and stimulus-specific manner. IL-17 and IL-22 drive MMP-3 but suppress MMP-9 in airway epithelium. The PI3Kinase/p110a/p70S6K pathway is a crucial target and its immuno-modulation (eg, rapamycin) is a
A

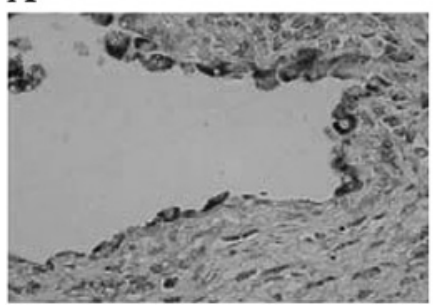

B

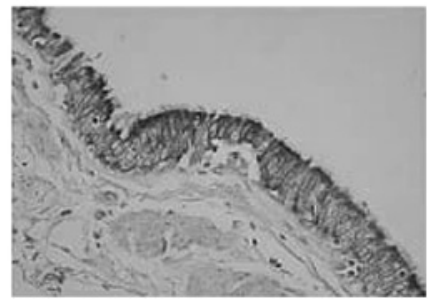

Abstract T1 Figure 1 Pulmonary epithelial cells around a TB granuloma expressed IL-17 (A). Strong immunoreactivity for MMP-3 (B) in pulmonary epithelial cells around TB granulomas was also detected. Control lungs (not shown) were negative. potential adjunctive therapy to limit tissue destruction and shorten chemotherapy in TB.

\section{T2 SINGLE NUCLEOTIDE POLYMORPHISMS IN THE FICOLIN-2 GENE PREDISPOSE TO PSEUDOMONAS AERUGINOSA INFECTION AND DISEASE SEVERITY IN NON-CYSTIC FIBROSIS BRONCHIECTASIS}

doi:10.1136/thoraxjnl-2011-201054a.2

${ }^{1} \mathrm{~J}$ D Chalmers, ${ }^{2} \mathrm{D}$ C Kilpatrick, ${ }^{1} \mathrm{~B} \mathrm{~J}$ McHugh, ${ }^{1} \mathrm{M}$ P Smith, ${ }^{1} \mathrm{~J}$ R W Govan, ${ }^{1} \mathrm{C}$ Doherty, ${ }^{3} \mathrm{M}$ Matsushita, ${ }^{4} \mathrm{~S} P$ Hart, ${ }^{5} \mathrm{~T}$ Sethi, ${ }^{1} \mathrm{~A}$ T Hill. ${ }^{1}$ University of Edinburgh, Edinburgh, UK ${ }^{2}$ Scottish National Blood Transfusion Service, Edinburgh, UK; ${ }^{3}$ Tokai University, Kanagawa, UK; ${ }^{4}$ University of Hull, Hull, UK; ${ }^{5}$ King College, London, UK

Introduction Bronchiectasis is associated with a destructive cycle of bacterial infection, airway inflammation and airway structural damage. Ficolins are a family of recently described serum pattern recognition molecules capable of binding to micro-organisms and activating complement through $\mathrm{MBL}$ associated serine protease-2 (MASP-2). Their role in chronic lung disease has not previously been investigated.

Methods Serum levels of Ficolin-2 and MASP-2 were determined in 470 patients with idiopathic non-cystic fibrosis bronchiectasis and 400 matched control subjects by ELISA. Single nucleotide polymorphisms were determined using TaqMan PCR based genotyping. Bacterial binding was determined using ELISA and flow cytometry. Neutrophils from healthy donors were isolated by percoll gradient centrifugation and used in phagocytosis assays with FITC-labelled Pseudomonas aeruginosa strain PA01.

Results Genotyping success was $>95 \%$ and all SNP's were in Hardy-Weinburg equilibrium $(p>0.05)$. Bronchiectasis was associated with a homozygous mutation in the promoter of ficolin-2 (rs3124952) (28.5\% vs $19.5 \%, \mathrm{p}=0.002)$ and a homozygous mutation in exon-8 of the ficolin-2 gene causing impaired binding (rs 17549193$)(12 \%$ vs $8.4 \%, p=0.04)$. Low serum levels $(<1.6 \mu \mathrm{g} / \mathrm{ml}$, $2 \mathrm{SD}$. below the mean for controls) were strongly associated with the promoter polymorphisms $(\mathrm{p}<0.0001)$ and with bronchiectasis $(18.7 \%$ vs $7.8 \%, p<0.0001)$. In-vitro, ficolin-2 bound to over 60 strains of $P$ aeruginosa, including clinical isolates and mucoid strains, activating the lectin pathway of complement and promoting C3 and C4 deposition. Ficolin-2 also bound to Streptococcus pneumoniae,
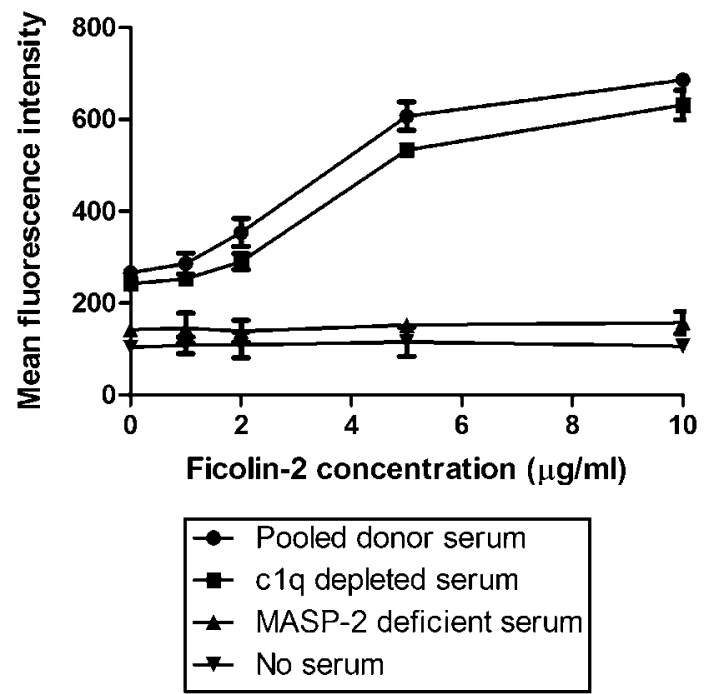

Abstract T2 Figure 1 Phagocytosis of FITC labelled Pseudomonas aeruginosa by neutrophils is enhanced by recombinant Ficolin-2 in the presence of complement. 